\title{
BMJ Open Sleep duration and mortality in the elderly: a systematic review with meta-analysis
}

Andressa Alves da Silva, ${ }^{1}$ Renato Gorga Bandeira de Mello,
Camila Wohlgemuth Schaan, ${ }^{2}$ Flávio D Fuchs, ${ }^{3}$ Susan Redline, ${ }^{4}$ Sandra C Fuchs ${ }^{1,2}$

To cite: Silva AA, Mello RB, Schaan CW, et al. Sleep duration and mortality in the elderly: a systematic review with meta-analysis. BMJ Open 2016;6:e008119. doi:10.1136/bmjopen-2015008119

- Prepublication history for this paper is available online. To view these files please visit the journal online (http://dx.doi.org/10.1136/ bmjopen-2015-008119).

Received 6 March 2015 Revised 6 November 2015 Accepted 14 December 2015

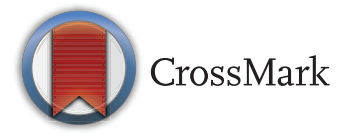

For numbered affiliations see end of article.

Correspondence to

Professor Sandra C Fuchs; sfuchs@hcpa.edu.br

\section{ABSTRACT}

Objective: The purpose of our study was to evaluate the association between short and long sleep duration and all-cause and cardiovascular mortality among elderly individuals.

Design: Systematic review and meta-analysis of population-based cohort studies.

Setting: Articles were retrieved from international and national electronic databases.

Study selection: Studies were identified in PubMed, EMBASE, LILACS (Latin American and Caribbean Health Sciences Literature), IBECS (Bibliographic Index on Health Sciences from Spain) and CAPES (PhD thesis repository) between 1980 and 2015. Studies which met all criteria were eligible: participants aged 60 years or over, assessment of sleep duration as $24 \mathrm{~h}$, nighttime or daytime sleep, evaluation of all-cause or cause-specific mortality, population-based cohort studies conducted on representative samples. There was no language restriction and studies published as abstracts were excluded.

Data extraction: Data were analysed using the Comprehensive Meta-Analysis software (V.3.3.070), and summary estimates (relative risk (RR), $95 \% \mathrm{Cl}$ ) were calculated using a random effects model. Heterogeneity and consistency were evaluated through Cochran's $Q$ and the $I^{2}$ statistics, respectively, and sensitivity analyses were conducted.

Primary and secondary outcome measures: All-cause and cardiovascular mortality.

Results: Overall, 27 cohort studies were selected, comprising >70 000 elderly individuals, and followed up from 3.4 to 35 years. In the pooled analysis, long and short sleep duration were associated with increased all-cause mortality (RR 1.33; 95\% Cl 1.24 to 1.43 and RR 1.07; 95\% Cl 1.03 to 1.11 , respectively), compared with the reference category. For cardiovascular mortality, the pooled relative risks were $1.43(95 \% \mathrm{Cl} 1.15$ to 1.78$)$ for long sleep, and 1.18 (95\% Cl 0.76 to 1.84) for short sleep. Daytime napping $\geq 30$ min was associated with risk of allcause mortality (RR $1.27 ; 95 \% \mathrm{Cl} 1.08$ to 1.49 ), compared with no daytime sleep, but longer sleep duration ( $\geq 2.0 \mathrm{~h}$ ) was not (RR 1.34; 95\% Cl 1.95 to 1.90$)$.

Conclusions: Among elderly individuals, long and short sleep duration are associated with increased risk

\section{Strengths and limitations of this study}

- Long and short sleep duration are associated with increased risk for all-cause mortality, but only long sleep duration is significantly associated with cardiovascular mortality.

- The definition of sleep duration varied among studies.

- There is scarce evidence about mechanisms to explain the association.

- Sleep fragmentation is hardly accessed and is not available in most of the studies.

- Meta-analysis of observational data cannot fully control for confounding factors.

for all-cause mortality. Long sleep duration is associated with cardiovascular mortality.

\section{INTRODUCTION}

The association between sleep duration and a range of health outcomes has received growing attention. Laboratory and epidemiological-based evidence demonstrate that self-reported short and long sleep duration (often defined as $<6$ or 7 and $>8$ or $9 \mathrm{~h}$, respectively) are associated with cardiovascular disease (CVD) ${ }^{1-6}$ diabetes mellitus, $^{7-10}$ obesity $^{11-15}$ and poor self-rated health. $^{16}$ In addition, large cohort studies $^{5}$ 17-19 and systematic reviews 2020 have shown that sleep duration is associated with increased risk of mortality among middle-aged adults.

Health-related sleeping problems are particularly relevant for individuals aged 60 years or older, a group which is expected to reach two billion in $2050 .^{22}$ Sleep problems are prevalent in the elderly population and approximately $50 \%$ have sleep-related symptoms. ${ }^{23}$ The association between sleep duration and mortality has scarcely been 
investigated in this population, with reports that have been inconsistent and based on limited sample sizes. $^{21} 2425$

The lack of statistical power is overcome by carrying out meta-analyses, which allows that results from different studies can be combined, increasing the statistical power and precision of estimates. ${ }^{8}$ In this systematic review with meta-analysis, we aimed to summarise the evidence from population-based cohort studies that addresses the association between short or long sleep duration and mortality in elderly individuals.

\section{METHODS}

This study was carried out in accordance with the recommendations of the Transparent Reporting of Systematic Reviews and Meta-Analyses (PRISMA) guideline. ${ }^{26}$

\section{Literature search}

Retrieval of articles was based on Medical Subject Headings (MeSH) and Health Sciences Descriptors (DeCS) descriptors, which were combined using Boolean operators (table 1). We searched the electronic databases PubMed, EMBASE, LILACS (Latin American and Caribbean Health Sciences Literature) and IBECS (Bibliographic Index on Health Sciences from Spain). We also searched reference lists of original and review articles. No language restrictions were applied.

\section{Inclusion and exclusion criteria}

Eligible studies were selected among those published between 1980 and 2015, which met all of the following criteria: (1) participants aged 60 years or over; (2) assessment of sleep duration as $24 \mathrm{~h}$, nighttime or daytime sleep; (3) evaluation of all-cause or cause-specific mortality and (4) population-based cohort studies conducted on representative samples. Studies published as abstracts were excluded, as well as thesis or dissertations that were also published as articles. If multiple published reports from the same study were available, we included the one with the most detailed information for exposure and outcome. The systematic review did not require ethical approval.

\section{Study selection and data extraction}

Study selection was conducted in two stages: an initial screening of titles and abstracts to identify potentially relevant papers, followed by screening of the full-length papers. Two independent reviewers (AAdS and CWS) assessed eligibility using a standardised protocol (available under request). The assessors appraised all studies for internal validity, using the Strengthening the Reporting of Observational Studies in Epidemiology (STROBE) statement, and a qualitative assessment of its items was held: ascertainment of population, exposure, comparison group, outcome of interest that was not present at the start of the study (which is obvious since the outcome is death), comparability of cohorts on the basis of the design or analysis, assessment of outcome by independent blind assessment (for CVD deaths), follow-up long enough for outcomes to occur, and adequacy of follow-up of the cohort studies. We found that all studies provided enough information to ensure the quality of reporting.

Data were extracted using a pretested spreadsheet. Agreement beyond chance among reviewers (interassessor reliability) was assessed by the $\mathrm{\kappa}$ statistic at the pilot phase, and subsequent disagreements were resolved by consensus with an independent reviewer (RGBdM). The extraction of data included information to identify the authors, population (place, time and demographic characteristics), study design, year of publication, baseline and follow-up periods, number and cause of deaths, types of sleep ( $24 \mathrm{~h}$ sleep, nighttime sleep or daytime sleep), measurement of sleep duration, categories of 'short' and 'long' sleep duration, reference category used in the analysis, outcomes assessment, point estimate used in the studies-relative risk (RR) or HR-and 95\% CI, and covariates used in the multivariate analysis.

\section{Table 1 Search strategy used to locate articles in the database of MEDLINE/PubMed* \\ Participants Aged $\geq 60$ years aged [mesh terms] OR aged [all fields] OR geriatrics [mesh terms] OR geriatrics [all fields] OR elderly [all fields] OR 'older age' [all fields] OR 'older adult' [all fields] OR 'older adults' [all fields] OR 'older person' [all fields] OR 'older people' [all fields] OR 'older man' [all fields] OR 'older men' [all fields] OR 'older woman' [all fields] OR 'older women' [all fields] OR aging [mesh terms] OR aging [all fields] OR ageing [all fields] \\ Exposure Sleep duration sleep [mesh terms] OR sleep [all fields] OR sleep disorders [mesh terms] OR 'sleep disorders' [all fields] OR 'sleep duration' [all fields] OR insomnia [all fields] OR drowsiness [all fields] OR drowse [all fields] OR napping [all fields] OR nap [all fields] OR naps [all fields] OR siesta [all fields] \\ Outcome Mortality mortality [mesh terms] OR mortality [all fields] OR death [mesh terms] OR death [all fields] OR deaths [all fields] \\ Design Cohort studies cohort studies [mesh terms] OR cohort [all fields] \\ Filters Publication date 01/01/1980-31/08/2015}

* Similar strategies were used to perform the search in the EMBASE, LILACS (Latin American and Caribbean Health Sciences Literature) and IBECS (Bibliographic Index on Health Sciences from Spain) databases. 


\section{Statistical analysis}

Studies were assessed for selection, information, measurement and confounding biases, based on the STROBE guideline. ${ }^{27}$ Summary estimates (RR, 95\% CI) were calculated using the random effects model for short and long sleep categories. The RR and 95\% CI were extracted for all-cause and cardiovascular mortality, and adjusted estimates were presented in preference to crude estimates. Results, reported separately for men and women or age groups, were entered into the analysis as different cohorts. For articles reporting multiple categories of short and long sleep duration (eg, <6.0 and 6.0-6.9 for short sleep; 8.0-9.9 and $\geq 10 \mathrm{~h}$ for long sleep), the shortest and the longest categories (eg, $<6.0$ and $\geq 10.0 \mathrm{~h}$ for short and long sleep, respectively) were used. Heterogeneity and inconsistency index were evaluated through Cochran's $Q$ test and the $\mathrm{I}^{2}$ statistics, respectively, using the Comprehensive Meta-Analysis software (V.3.3.070).

Subgroup analysis was performed to assess sources of heterogeneity and to check for the potential impact of the following factors on the association between sleep duration and mortality: sleep duration assessment $(24 \mathrm{~h}$ or nighttime sleep), gender, geographic location, duration of follow-up, reference category and definition of sleep duration. Further, since there are several potential confounders and multivariate analyses were performed in individual studies, subgroup analyses were also conducted according to the adjusted variables. The influence of individual studies on the magnitude of the pooled estimate was examined by omitting one study at a time and examining the extent to which inferences were dependent on a particular study (sensitivity analysis).

Funnel plots were used to detect publication bias, and Egger's regression test was applied to measure the funnel plot asymmetry. Combined risks were recalculated after estimating from the asymmetry of the funnel plot the number of 'missing' studies and their effect sizes and SEs-a method known as 'trim and fill'. ${ }^{28}$

\section{RESULTS}

Overall, 27 cohort studies were selected (figure 1), comprising $>77000$ elderly individuals. Authors of studies, which also included participants younger than 60 years, whose corresponding author was available, were contacted to request a reanalysis of data, restricted to elderly individuals. Five authors ${ }^{29-33}$ did a reanalysis and sent additional data. Those studies were included in the meta-analysis.

Table 2 summarises characteristics of the studies from 13 different countries, including two studies that recruited only women, ${ }^{33} 34$ and one with men only. ${ }^{35}$ Six authors reported outcomes separately for men and women. $^{30} 32 \quad 36-39$ The number of participants in the studies varied from $167^{40}$ to $11395^{41}$ and the follow-up period ranged from $3.4^{35}$ to $35^{29}$ years.

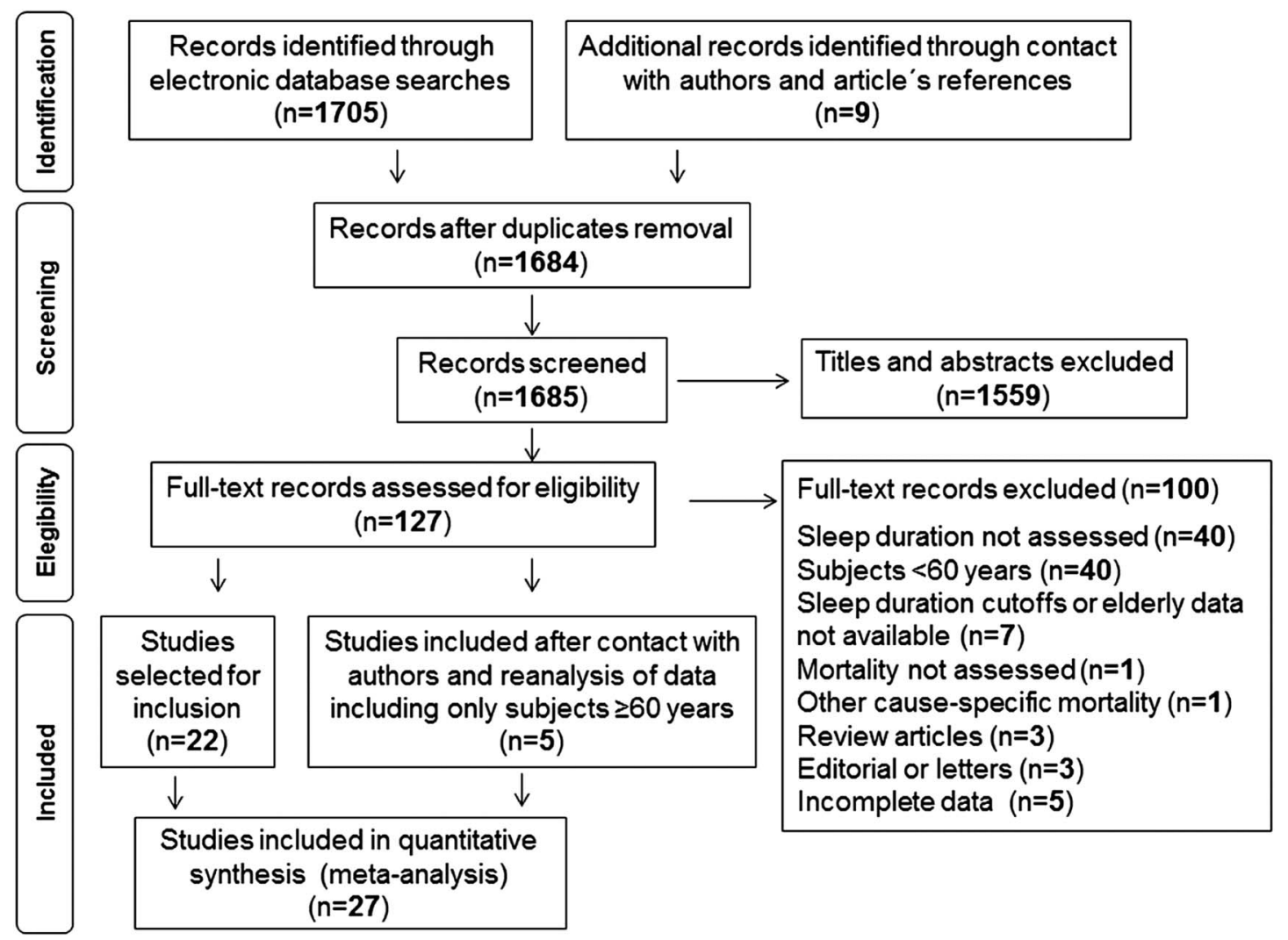

Figure 1 Flow chart of records retrieved, screened and included in the systematic review. 
Table 2 Description of the studies included in the meta-analysis (in order of baseline year)

\begin{tabular}{|c|c|c|c|c|c|c|c|}
\hline First author, publication year & $\begin{array}{l}\text { Baseline } \\
\text { year }\end{array}$ & Country & Age (years) & Sex & $\mathbf{N}$ & Deaths & $\begin{array}{l}\text { Follow-up } \\
\text { (years) }\end{array}$ \\
\hline Kaplan, $1987^{42}$ & 1965 & USA & $60-94$ & Men, women & 4174 & DNA & 17 \\
\hline Kronholm, $2011^{29}$ & 1972 & Finland & $60-64$ & Men, women & 1210 & 1065 & 35 \\
\hline Gale, $1998^{43}$ & 1973 & UK & $\geq 65$ & Men, women & 1229 & 1158 & 23 \\
\hline Branch, $1984^{44}$ & 1974 & USA & $\overline{66}-98$ & Men, women & 1235 & 275 & 5 \\
\hline Gangwisch, $2008^{45}$ & 1982 & USA & $60-86$ & Men, women & 3983 & 1877 & $8-10$ \\
\hline Mallon, $2002^{30}$ & 1983 & Sweden & $60-65$ & Men, women & 338 & 91 & 12 \\
\hline Jung, $2013^{39}$ & 1984 & USA & $69-96$ & Men, women & 2001 & 1224 & 19 \\
\hline Burazeri, $2003^{36}$ & 1985 & Israel & $\geq 65$ & Men, women & 828 & 276 & 10 \\
\hline Rumble, $1992^{46}$ & 1985 & UK & $\geq 65$ & Men, women & 1042 & 352 & 5 \\
\hline Ruigomez, $1995^{47}$ & 1986 & Spain & $65-97$ & Men, women & 989 & 224 & 5 \\
\hline Stone, $2009^{34}$ & 1986 & USA & $\geq 69$ & Women & 8101 & 1922 & 6.9 \\
\hline Cohen-Mansfield, $2012^{48}$ & 1989 & Israel & $75-94$ & Men, women & 1166 & DNA & 20 \\
\hline Kim, $2013^{49}$ & 1990 & USA & $\geq 65$ & Men, women & DNA & 4103 & 12.9 \\
\hline Seki, $2001^{50}$ & 1990 & Japan & $60-74$ & Men, women & 1065 & 123 & 7.5 \\
\hline Lan, $2007^{37}$ & 1993 & Taiwan & $\geq 64$ & Men, women & 3079 & 1338 & 8.4 \\
\hline Leng, $2014^{51}$ & 1993 & UK & $>65$ & Men, women & 3378 & 2464 & 13 \\
\hline Yeo, $2013^{52}$ & 1993 & Korea & $\geq 60$ & Men, women & 5538 & 1223 & 9.4 \\
\hline Kakizaki, $2013^{31}$ & 1994 & Japan & $\geq 70$ & Men, women & 9690 & 3960 & 10.8 \\
\hline Werle, $2011^{40}$ & 1994 & Brazil & $\geq 80$ & Men, women & 167 & 122 & 8.7 \\
\hline Kripke, $2011^{33}$ & 1995 & USA & $60-81$ & Women & 355 & 79 & 10.5 \\
\hline Castro-Costa, $2011^{24}$ & 1997 & Brazil & $63-75$ & Men, women & 1512 & 440 & 7.5 \\
\hline Chen, $2013^{53}$ & 1999 & Taiwan & $>65$ & Men, women & 4064 & 1004 & 9 \\
\hline Suzuki, $2009^{41}$ & 1999 & Japan & $65-85$ & Men, women & 11395 & 1004 & 5.4 \\
\hline Ensrud, $2012^{35}$ & 2000 & USA & $\geq 65$ & Men & 2505 & 180 & 3.4 \\
\hline Mesas, $2010^{54}$ & 2000 & Spain & $\geq 60$ & Men, women & 3820 & 897 & 6.8 \\
\hline Lee, $2014^{38}$ & 2001 & China & $>65$ & Men, women & 3427 & 24 & 5 \\
\hline Zuurbier, $2015^{32}$ & 2004 & Holland & $60-98$ & Men, women & 1073 & 142 & 7.3 \\
\hline
\end{tabular}

Table 3 summarises additional characteristics, including definitions of 'short' and 'long' sleep duration, which were adopted in the studies, and describes the methods used in each study. All-cause mortality was reported in 27 studies $^{24} 29-54$ and cardiovascular mortality in 9 studies. $^{29} 3134363740415253$ Nighttime sleep duration was reported in 16 studies, ${ }^{24} \begin{array}{llllll}29 & 30 & 33 & 34 & 370\end{array}$ $42-46 \quad 48 \quad 53$ and $24 \mathrm{~h}$ sleep in 11 studies, ${ }^{31} 32 \quad 34$ 3540414749505254 while daytime sleep was evaluated in 4 studies. ${ }^{36} \quad 37 \quad 45 \quad 51$ Sleep duration was assessed by self-report using a single survey question in almost all studies, and cause of death was based on death certificates, although a few studies used additional sources of information. ${ }^{24} 2932 \quad 33 \quad 35 \quad 38 \quad 40 \quad 44 \quad 46 \quad 52$ One included study assessed sleep duration objectively by actigraphy. ${ }^{35}$ Kripke $e t a l^{33}$ and Zuurbier et $a l^{32}$ assessed sleep duration using a questionnaire in addition to actigraphic studies.

Eighteen of the 27 studies adjusted for or stratified for age and sex in the analyses. ${ }^{30-32} 36-40414345 \quad 47-50 \quad 52-54$ Seventeen studies adjusted for demographic and lifestyle characteristics plus comorbid conditions, ${ }^{24} \quad 30-32 \quad 34 \quad 35$ 37-39 41 45 48 49 51-54 and 10 included sleep-related medications as confounders. ${ }^{24} \quad 30 \quad 32 \quad 34 \quad 35 \quad 38 \quad 46 \quad 51 \quad 53 \quad 54$ Only two studies adjusted for functional status (activities of daily living) 4854 and frailty. ${ }^{35} 38$

\section{Long sleep duration and mortality}

Figure 2 shows that long sleep duration was associated with a greater risk of death (RR 1.33; 95\% CI 1.24 to 1.43), with moderate heterogeneity among studies $\left(\mathrm{I}^{2}=61.9 \%, \mathrm{Q}=73.55, \mathrm{p}<0.001\right.$; random model $)$. Despite the evidence of publication bias, the adjusted effect provided by the trim and fill method remained statistically significant (RR 1.27; 95\% CI 1.18 to 1.36). Sensitivity analysis, by omitting one study at a time, showed similar results with no statistically significant differences. However, the removal of the study performed by Mallon $e t a b^{30}$ reduced the risk of long sleep duration on mortality to RR 1.22 (95\% CI 1.18 to 1.26$)$.

The association between long duration of sleep and cardiovascular mortality was also statistically significant (RR $1.43 ; 95 \%$ CI 1.15 to 1.78 ). Daytime napping $\geq 30 \mathrm{~min}$ was associated with risk of all-cause mortality (RR 1.27; 95\% CI 1.08 to 1.49), compared with no daytime sleep. However, in separate analysis, longer sleep duration $(\geq 2.0 \mathrm{~h})$ was not significantly associated with risk of death (RR 1.34; $95 \%$ CI 1.95 to 1.90 ).

\section{Short sleep duration and mortality}

Figure 3 shows the association between short sleep and all-cause mortality. Pooled analysis showed that short sleep duration was significantly associated with risk of 
Table 3 Characteristics of the studies included in the meta-analysis (in order of baseline year)

\begin{tabular}{|c|c|c|c|}
\hline $\begin{array}{l}\text { First author, } \\
\text { publication year }\end{array}$ & $\begin{array}{l}\text { Ascertainment of } \\
\text { sleep (ref vs } \\
\text { exposure) }\end{array}$ & $\begin{array}{l}\text { Ascertainment of } \\
\text { mortality }\end{array}$ & Adjustment for confounders \\
\hline Kaplan, $1987^{42}$ & $\begin{array}{l}\text { Questionnaire } \\
\text { Nighttime sleep } \\
7.0-8.0 \text { vs }<7.0 \mathrm{~h} \\
7.0-8.0 \mathrm{vs}>8.0 \mathrm{~h}\end{array}$ & $\begin{array}{l}\text { Death certificate } \\
\text { All cause }\end{array}$ & Age, self-reported health status \\
\hline Kronholm, $2011^{29}$ & $\begin{array}{l}\text { Questionnaire } \\
\text { Nighttime sleep } \\
7.0-8.0 \text { vs } \leq 5.0 \mathrm{~h} \\
7.0-8.0 \text { vs } \geq 10.0 \mathrm{~h}\end{array}$ & $\begin{array}{l}\text { Death certificate and } \\
\text { hospital discharge } \\
\text { register } \\
\text { All cause and CVD }\end{array}$ & None \\
\hline Gale, $1998^{43}$ & $\begin{array}{l}\text { Questionnaire } \\
\text { Nighttime sleep } \\
9.0 \text { vs } \leq 7.0 \mathrm{~h} \\
9.0 \text { vs } \geq 12 \mathrm{~h}\end{array}$ & $\begin{array}{l}\text { Death certificate } \\
\text { All cause }\end{array}$ & $\begin{array}{l}\text { Age, sex, geriatrician's diagnoses of illness, social class, } \\
\text { systolic BP, BMI }\end{array}$ \\
\hline Branch, $1984^{44}$ & $\begin{array}{l}\text { Questionnaire } \\
\text { Nighttime sleep } \\
7.0-8.0 \text { vs } 1.0-6.0 \mathrm{~h} \\
7.0-8.0 \text { vs }>8 \mathrm{~h}\end{array}$ & $\begin{array}{l}\text { Death certificate } \\
\text { All cause }\end{array}$ & None \\
\hline $\begin{array}{l}\text { Gangwisch, } \\
2008^{45}\end{array}$ & $\begin{array}{l}\text { Questionnaire } \\
\text { Nighttime sleep } \\
7.0 \text { vs } \leq 5.0 \mathrm{~h} \\
7.0 \text { vs } \geq 9.0 \mathrm{~h}\end{array}$ & $\begin{array}{l}\text { Death certificate and } \\
\text { proxy interviews } \\
\text { All cause }\end{array}$ & $\begin{array}{l}\text { Age, physical activity, smoking, depression, sex, } \\
\text { education, living alone, low income, daytime sleepiness, } \\
\text { nighttime awakening, ethnicity, sleeping pill use, BMI, } \\
\text { diabetes, hypertension, general health, cancer }\end{array}$ \\
\hline Mallon, $2002^{30}$ & $\begin{array}{l}\text { Questionnaire } \\
\text { Nighttime sleep } \\
6.0-8.0 \text { vs }<6.0 \mathrm{~h} \\
6.0-8.0 \text { vs }>8.0 \mathrm{~h}\end{array}$ & $\begin{array}{l}\text { Death certificate } \\
\text { All cause }\end{array}$ & $\begin{array}{l}\text { Age, living alone, not being married, smoking, diabetes, } \\
\text { CVD, depression, insomnia, BMI, joint pain, respiratory } \\
\text { disease, gastrointestinal disease, habitual sleeping pills } \\
\text { use, habitual snoring }\end{array}$ \\
\hline Jung, $2013^{39}$ & $\begin{array}{l}\text { Questionnaire } \\
\text { Nighttime sleep } \\
7.0-7.9 \text { vs } 6.0 \mathrm{~h} \\
7.0-7.9 \mathrm{~h} \text { vs } \geq 9.0 \mathrm{~h} \\
\text { Daytime sleep } \\
\text { None vs } \geq 0.5 \mathrm{~h}\end{array}$ & $\begin{array}{l}\text { Death certificate } \\
(90 \%) \text {, or notice from } \\
\text { a family member or } \\
\text { published obituary } \\
(10 \%) \\
\text { All cause }\end{array}$ & $\begin{array}{l}\text { Age, nap duration, depression, exercise, education, } \\
\text { smoking, alcohol consumption, hypertension, diabetes, } \\
\text { coronary heart disease, stroke, cancer, sleep-related } \\
\text { medications and postmenopausal oestrogen }\end{array}$ \\
\hline Burazeri, $2003^{36}$ & $\begin{array}{l}\text { Questionnaire } \\
\text { Daytime sleep } \\
\text { None vs } \geq 2.0 \mathrm{~h}\end{array}$ & $\begin{array}{l}\text { Death certificate } \\
\text { All cause and CVD }\end{array}$ & Age \\
\hline Rumble, $1992^{46}$ & $\begin{array}{l}\text { Questionnaire } \\
\text { Nighttime sleep } \\
4.0-9.9 \text { vs }<4.0 \mathrm{~h} \\
4.0-9.9 \text { vs } \geq 10.0 \mathrm{~h}\end{array}$ & $\begin{array}{l}\text { Death certificate and } \\
\text { patient records } \\
\text { All cause }\end{array}$ & Sex, health 'risk', sleep medication \\
\hline $\begin{array}{l}\text { Ruigomez, } \\
1995^{47}\end{array}$ & $\begin{array}{l}\text { Questionnaire } \\
24 \mathrm{~h} \text { sleep } \\
7.0-9.0 \text { vs } \leq 5.0 \mathrm{~h} \\
7.0-9.0 \text { vs }>9.0 \mathrm{~h}\end{array}$ & $\begin{array}{l}\text { Death certificate } \\
\text { All cause }\end{array}$ & Age, sex, education, perceived health status \\
\hline Stone, $2009^{34}$ & $\begin{array}{l}\text { Questionnaire } \\
\text { Nighttime sleep } \\
6.0-8.0 \text { vs }<6.0 \mathrm{~h} \\
6.0-8.0 \text { vs }>8.0 \mathrm{~h} \\
24 \mathrm{~h} \text { sleep } \\
8.0-8.9 \mathrm{vs}<6.0 \mathrm{~h} \\
8.0-8.9 \mathrm{vs} \geq 10.0 \mathrm{~h}\end{array}$ & $\begin{array}{l}\text { Death certificate } \\
\text { All cause, CVD, } \\
\text { cancer and other }\end{array}$ & $\begin{array}{l}\text { Age, BMI, history of at least one medical condition } \\
\text { (diabetes, Parkinson's disease, dementia, COPD, } \\
\text { non-skin cancer and osteoarthritis), CVD, hypertension, } \\
\text { walks for exercise, alcohol use, smoking status, } \\
\text { depression, cognitive impairment, oestrogen use, } \\
\text { benzodiazepine use }\end{array}$ \\
\hline $\begin{array}{l}\text { Cohen-Mansfield, } \\
2012^{48}\end{array}$ & $\begin{array}{l}\text { Questionnaire } \\
\text { Nighttime sleep } \\
7.0-9.0 \text { vs }<7.0 \mathrm{~h} \\
7.0-9.0 \mathrm{vs}>9.0 \mathrm{~h}\end{array}$ & $\begin{array}{l}\text { Death certificate } \\
\text { All cause }\end{array}$ & $\begin{array}{l}\text { Age, sex, origin, marital status, education, income, had } \\
\text { children, number of medications, comorbidity, subjective } \\
\text { health, } A D L \text { and IADL limitations, cognitive difficulties, } \\
\text { depressed affect }\end{array}$ \\
\hline Kim, $2013^{49}$ & $\begin{array}{l}\text { Questionnaire } \\
24 \mathrm{~h} \text { sleep } \\
7.0 \mathrm{vs} \leq 5.0 \mathrm{~h} \\
7.0 \mathrm{vs} \geq 9.0 \mathrm{~h}\end{array}$ & $\begin{array}{l}\text { Death certificate } \\
\text { All cause }\end{array}$ & $\begin{array}{l}\text { Age, sex, ethnicity, education, marital status, } \\
\text { hypertension, diabetes, alcohol consumption, energy } \\
\text { intake, BMI, physical activity, hours spent daily watching } \\
\text { television, smoking }\end{array}$ \\
\hline
\end{tabular}


Table 3 Continued

\begin{tabular}{|c|c|c|c|}
\hline $\begin{array}{l}\text { First author, } \\
\text { publication year }\end{array}$ & $\begin{array}{l}\text { Ascertainment of } \\
\text { sleep (ref vs } \\
\text { exposure) }\end{array}$ & $\begin{array}{l}\text { Ascertainment of } \\
\text { mortality }\end{array}$ & Adjustment for confounders \\
\hline Seki, $2001^{50}$ & $\begin{array}{l}\text { Questionnaire } \\
24 \mathrm{~h} \text { sleep } \\
7.0 \text { vs }<6.0 \mathrm{~h} \\
7.0 \mathrm{vs} \geq 9.0 \mathrm{~h}\end{array}$ & $\begin{array}{l}\text { Death certificate } \\
\text { All cause }\end{array}$ & $\begin{array}{l}\text { Age, sex, medical history (cerebrovascular disease, CVD, } \\
\text { hypertension, diabetes, liver disease, renal disease, } \\
\text { malignant neoplasm) }\end{array}$ \\
\hline Lan, $2007^{37}$ & $\begin{array}{l}\text { Questionnaire } \\
\text { Nighttime sleep } \\
7.0-7.9 \text { vs }<7.0 \mathrm{~h} \\
7.0-7.9 \text { vs } \geq 10 \mathrm{~h} \\
\text { Daytime sleep } \\
\text { None vs } \geq 2.0 \mathrm{~h}\end{array}$ & $\begin{array}{l}\text { Death certificate } \\
\text { All cause, CVD and } \\
\text { cancer }\end{array}$ & $\begin{array}{l}\text { Age, marital status, monthly income, cigarette smoking, } \\
\text { alcohol consumption, BMI, exercise, disease history } \\
\text { (heart disease, stroke and cancer), depression, afternoon } \\
\text { nap duration }\end{array}$ \\
\hline Leng, $2014^{51}$ & $\begin{array}{l}\text { Questionnaire } \\
\text { Daytime sleep } \\
\text { None vs } \geq 1.0 \mathrm{~h}\end{array}$ & $\begin{array}{l}\text { Death certificate } \\
\text { All cause }\end{array}$ & $\begin{array}{l}\text { Age, sex, social class, educational level, marital status, } \\
\text { employment status, BMI, physical activity level, smoking } \\
\text { status, alcohol intake, depression, self-reported general } \\
\text { health, hypnotic drug use, antidepressant use, COPD } \\
\text { drug use, time spent in bed at night, self-reported } \\
\text { pre-existing diseases, sleep apnoea }\end{array}$ \\
\hline Yeo, $2013^{52}$ & $\begin{array}{l}\text { Questionnaire } \\
24 \mathrm{~h} \text { sleep } \\
7.0 \text { vs } \leq 5.0 \mathrm{~h} \\
7.0 \text { vs } \geq 10.0 \mathrm{~h}\end{array}$ & $\begin{array}{l}\text { Death certificate } \\
\text { All cause, CVD, } \\
\text { cancer, external } \\
\text { cause, respiratory } \\
\text { disease and other }\end{array}$ & $\begin{array}{l}\text { Sex, educational attainment, BMI, cigarette smoking, } \\
\text { alcohol consumption, hypertension, diabetes, CVD, } \\
\text { metabolic syndrome }\end{array}$ \\
\hline Kakizaki, $2013^{31}$ & $\begin{array}{l}\text { Questionnaire } \\
24 \mathrm{~h} \text { sleep } \\
7.0 \text { vs } \leq 6.0 \mathrm{~h} \\
7.0 \text { vs } \geq 10.0 \mathrm{~h}\end{array}$ & $\begin{array}{l}\text { Death certificate } \\
\text { All cause, CVD, } \\
\text { cancer and other }\end{array}$ & $\begin{array}{l}\text { Age, sex, total caloric intake, BMI, marital status, } \\
\text { education, job status, history of myocardial infarction, } \\
\text { history of cancer, history of stroke, hypertension, } \\
\text { diabetes, smoking, alcohol consumption, time spent } \\
\text { walking, perceived mental stress, self-rated health }\end{array}$ \\
\hline Werle, $2011^{40}$ & $\begin{array}{l}\text { Questionnaire } \\
\text { Nighttime } \\
24 \mathrm{~h} \text { sleep } \\
\leq 8.0 \text { vs }>8.0 \mathrm{~h}\end{array}$ & $\begin{array}{l}\text { Death certificate, } \\
\text { proxy interviews and } \\
\text { patient records } \\
\text { All cause and CVD }\end{array}$ & $\begin{array}{l}\text { Age, sex, education, number of family members living } \\
\text { with the elderly, smoking, physical activity, BMI }\end{array}$ \\
\hline Kripke, $2011^{33}$ & $\begin{array}{l}\text { Questionnaire and } \\
\text { actigraphy } \\
\text { Nighttime sleep } \\
7.0 \text { vs } \leq 5.0 \mathrm{~h} \\
7.0 \text { vs } \geq 9.0 \mathrm{~h}\end{array}$ & $\begin{array}{l}\text { Proxy interviews and } \\
\text { social security death } \\
\text { index } \\
\text { All cause }\end{array}$ & None \\
\hline $\begin{array}{l}\text { Castro-Costa, } \\
2011^{24}\end{array}$ & $\begin{array}{l}\text { Questionnaire } \\
\text { Nighttime sleep } \\
7.0-7.9 \text { vs }<6.0 \mathrm{~h} \\
7.0-7.9 \text { vs } \geq 9.0 \mathrm{~h}\end{array}$ & $\begin{array}{l}\text { Death certificate and } \\
\text { proxy interviews } \\
\text { All cause }\end{array}$ & $\begin{array}{l}\text { Age, schooling, marital status, working status, education, } \\
\text { alcohol consumption, coffee consumption, smoking, } \\
\text { physical exercises, depressive symptoms, cognitive } \\
\text { functioning, psychoactive medications, physical } \\
\text { functioning, arthritis ascertainment, systolic BP, HDL } \\
\text { cholesterol ratio, diabetes, BMI }\end{array}$ \\
\hline Chen, $2013^{53}$ & $\begin{array}{l}\text { Questionnaire } \\
\text { Nighttime sleep } \\
7.0 \mathrm{vs} \leq 4.0 \mathrm{~h} 7.0 \mathrm{vs} \\
\geq 9.0 \mathrm{~h}\end{array}$ & $\begin{array}{l}\text { Death certificate } \\
\text { All cause and CVD }\end{array}$ & $\begin{array}{l}\text { Age, sex, living status, marital status, education, BMI, } \\
\text { insomnia, excessive daytime sleepiness, pain, smoking, } \\
\text { alcohol drinking, snorers, diabetes, hypertension, CVD, } \\
\text { stroke, gouty arthritis, depression, hypnotics, total sleep } \\
\text { time }\end{array}$ \\
\hline Suzuki, $2009^{41}$ & $\begin{array}{l}\text { Questionnaire } \\
24 \mathrm{~h} \text { sleep } \\
7.0 \text { vs } \leq 5.0 \mathrm{~h} \\
7.0 \text { vs } \geq 10.0 \mathrm{~h}\end{array}$ & $\begin{array}{l}\text { Death certificate } \\
\text { All cause and CVD }\end{array}$ & $\begin{array}{l}\text { Age, sex, BMI, smoking status, alcohol consumption, } \\
\text { frequency of physical activity, socioeconomic status, } \\
\text { mental health, hypertension, diabetes }\end{array}$ \\
\hline Ensrud, $2012^{35}$ & $\begin{array}{l}\text { Actigraphy } \\
24 \mathrm{~h} \text { sleep } \\
>5.0 \text { vs } \leq 5.0 \mathrm{~h}\end{array}$ & $\begin{array}{l}\text { Death certificate and } \\
\text { proxy interviews } \\
\text { All cause }\end{array}$ & $\begin{array}{l}\text { Age, race, site, health status, BMI, education, social } \\
\text { support, alcohol intake, smoking, antidepressant, } \\
\text { benzodiazepine, non-benzodiazepine sedative hypnotic } \\
\text { use, medical conditions, cognition, baseline frailty status }\end{array}$ \\
\hline
\end{tabular}


Table 3 Continued

\begin{tabular}{|c|c|c|c|}
\hline $\begin{array}{l}\text { First author, } \\
\text { publication year }\end{array}$ & $\begin{array}{l}\text { Ascertainment of } \\
\text { sleep (ref vs } \\
\text { exposure) }\end{array}$ & $\begin{array}{l}\text { Ascertainment of } \\
\text { mortality }\end{array}$ & Adjustment for confounders \\
\hline Mesas, $2010^{54}$ & $\begin{array}{l}\text { Questionnaire } \\
24 \mathrm{~h} \text { sleep } \\
7.0 \mathrm{vs} \leq 5.0 \mathrm{~h} \\
7.0 \mathrm{vs} \geq 11.0 \mathrm{~h}\end{array}$ & $\begin{array}{l}\text { Death certificate } \\
\text { All cause }\end{array}$ & $\begin{array}{l}\text { Age, sex, BMI, educational level, municipality of } \\
\text { residence, physical activity, smoking, alcohol } \\
\text { consumption, coffee consumption, social links, perceived } \\
\text { health, MMSE score, depression, Medical Outcomes } \\
\text { Study 36-item Short Form Survey Physical and Mental } \\
\text { Component Summary scores, instrumental activity of } \\
\text { daily living limitations, hypertension, ischaemic heart } \\
\text { disease, stroke, diabetes, cancer, COPD, Parkinson's } \\
\text { disease, arousal from sleep at night, use of anxiolytic } \\
\text { medication }\end{array}$ \\
\hline Lee, $2014^{38}$ & $\begin{array}{l}\text { Questionnaire } \\
\text { Nighttime sleep } \\
<10.0 \text { vs } \geq 10.0 \mathrm{~h}\end{array}$ & $\begin{array}{l}\text { Death certificate } \\
\text { All cause }\end{array}$ & $\begin{array}{l}\text { Age, smoking, mood, overweight (BMl>23), medical } \\
\text { conditions (diabetes, heart disease, history of cancer, } \\
\text { COPD, history of stroke), frailty }\end{array}$ \\
\hline Zuurbier, $2015^{32}$ & $\begin{array}{l}\text { Questionnaire and } \\
\text { actigraphy } \\
24 \mathrm{~h} \text { sleep } \\
6.0-7.5 \text { vs }<6.0 \mathrm{~h} \\
6.0-7.5 \text { vs }>7.5 \mathrm{~h}\end{array}$ & $\begin{array}{l}\text { Death certificate and } \\
\text { patient records } \\
\text { All cause }\end{array}$ & $\begin{array}{l}\text { Age, sex, ADL, smoking, diabetes, myocardial infarction, } \\
\text { stroke, cognitive functioning, depressive symptoms, BMI, } \\
\text { sleep medication, napping, apnoea }\end{array}$ \\
\hline
\end{tabular}

death (RR 1.07; 95\% CI 1.03 to 1.11 ). There was no evidence of publication bias $(\mathrm{p}=0.45)$ or heterogeneity among studies $\left(\mathrm{I}^{2}=4.7 \%, \mathrm{Q}=27.28, \mathrm{p}=0.39\right.$; fixed model $)$.
The trim and fill method detected three missing studies, but the revised estimate remained the same. Sensitivity analysis, by omitting one study at a time, showed similar

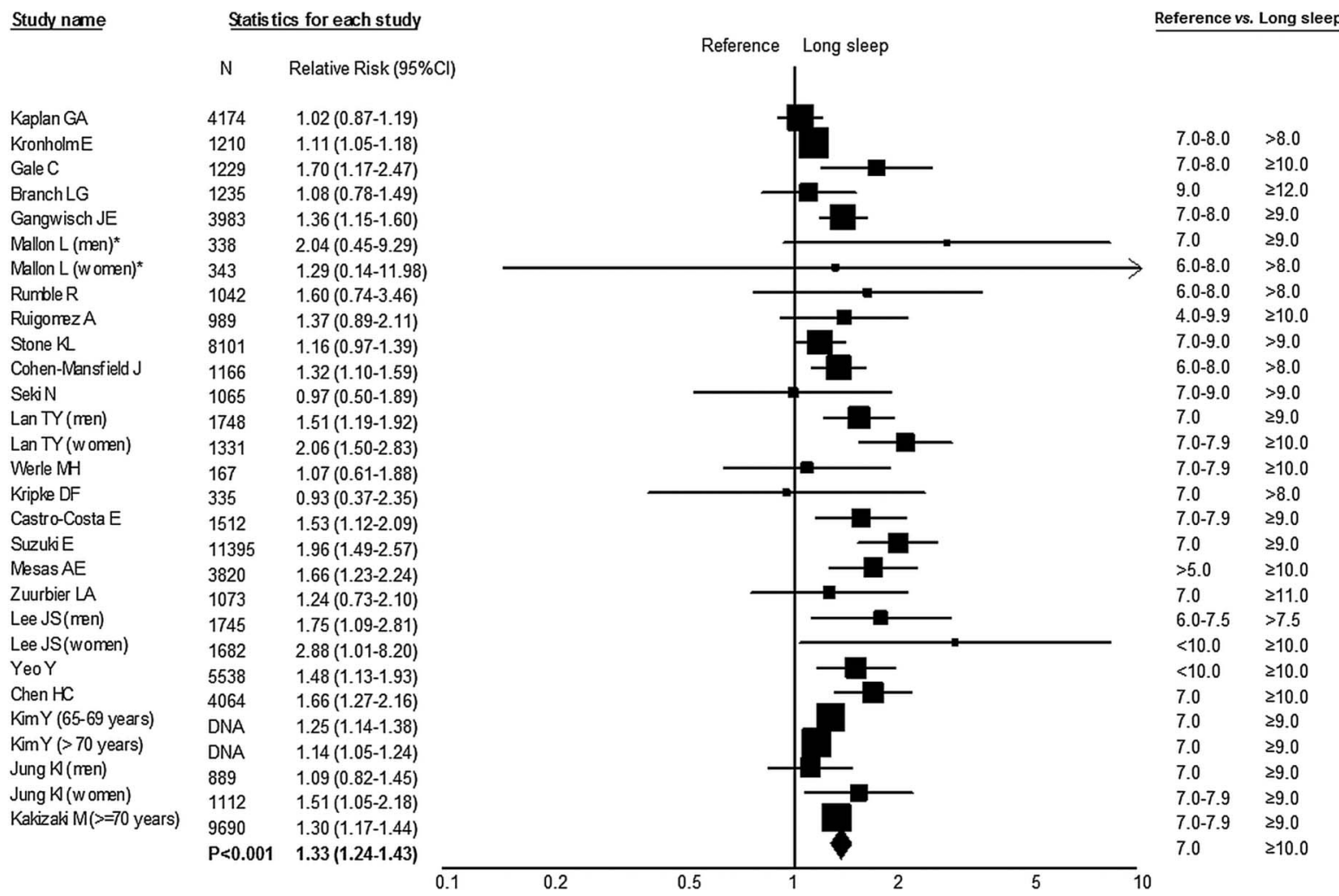

Heterogeneity: $L^{2}=61.9 \% ; Q=73.55 ; P<0.001$

Publication bias: Egger's test: $P=0.002$

Figure 2 Forest plot of mortality risk associated with long sleep duration compared with reference group. DNA, data not available. 
Study name

Statistics for each study

N Relative Risk $(95 \% \mathrm{Cl})$

Kronholm E

Gale C

Branch LG

Gangwisch JE

Mallon $L$ (men)

Mallon $L$ (women)

Rumble $\mathrm{R}$

Ruigomez A

Stone KL

Cohen-Mansfield JE

Seki N

Lan TY (men)

Lan TY (women)

Kripke DF

Castro-Costa E

Suzuki E

Ensrud KE

Mesas AE

Zuurbier LA*

YeoY

Chen $\mathrm{HC}$

Kim Y (65-69 years)

Kim $Y$ (> 70 years)

Jung KI (men)

Jung KI (women)

Kakizaki M

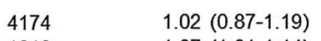

$1210 \quad 1.07(1.01-1.14)$

$1229 \quad 1.00(0.71-1.41)$

$1235 \quad 1.08(0.78-1.49)$

$3983 \quad 1.27(1.06-1.53)$

$338 \quad 0.05(0.00-0.48)$

$343 \quad 0.35(0.03-4.17)$

$1042 \quad 1.12(0.47-2.68)$

$989 \quad 0.83(0.56-1.23)$

$1.02(0.87-1.19)$

$0.98(0.85-1.14)$

$0.98(0.85-1.14)$
$1.74(0.72-4.22)$

$0.98(0.76-1.26)$

$1.14(0.77-1.68)$

$0.83(0.40-1.73)$

1.09 (0.78-1.53)

1.08 (0.65-1.80)

$0.92(0.66-1.28)$

$1.42(1.03-1.95)$

$1.12(0.75-1.68)$

$1.23(1.03-1.47)$

1.00 (0.75-1.33)

$1.13(1.02-1.26)$

$1.09(0.99-1.20)$

$0.98(0.67-1.43)$

$1.11(0.77-1.60)$

$0.98(0.87-1.10)$

1.07 (1.03-1.11)

Heterogeneity: $\left.\right|^{2}=4.7 \% ; Q=27.28 ; P=0.39$

Publication bias: Egger's test: $P=0.45$

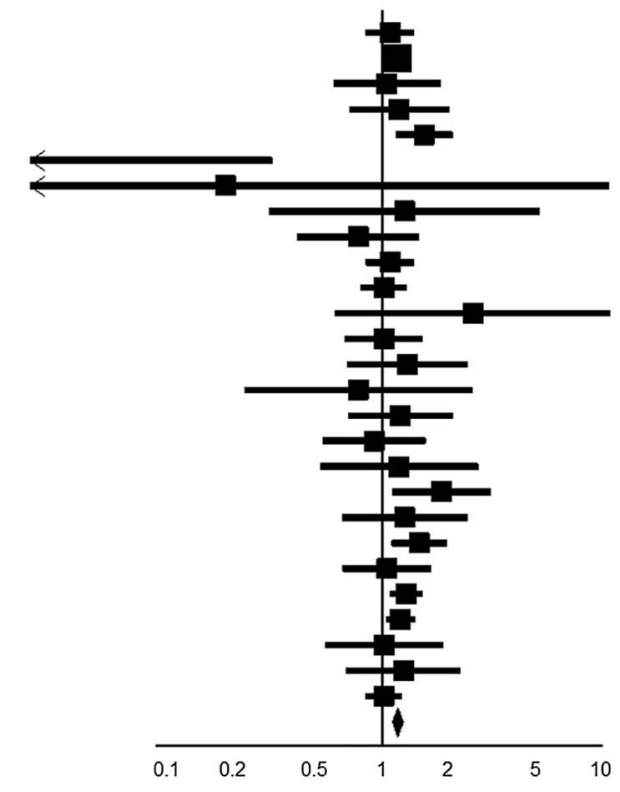

Reference Short sleep

Figure 3 Forest plot of mortality risk associated with short sleep duration compared with reference group. DNA, data not available.

results with no statistically significant differences. Pooled analysis remained statistically significant after excluding Kronholm et $a l \mathrm{~s}^{29}$ study, which had the largest influence (relative weight: $31.6 \%$ ) in the overall pooled estimate, as well as with the exclusion of Mallon et $a l \mathrm{~s}^{30}$ study. The association between short duration of sleep and cardiovascular mortality was not statistically significant (RR $1.18 ; 95 \%$ CI 0.76 to 1.84 ).

\section{Sources of heterogeneity}

The risk due to the long duration of sleep was detected for nighttime sleep and $24 \mathrm{~h}$, between men and women in cohorts conducted in America, Europe and Asia, and for different definitions of long sleep (figure 4A). It progressively increased as the definition of long sleep changed from $>8.0$ to $\geq 10.0 \mathrm{~h}$. The association tended to be weaker in studies carried out in American countries compared with Asian population studies. There was a stronger effect for studies with a length of follow-up $\leq 10$ years in analysis of long sleep. In subgroup analysis of short sleep duration, the effect did not differ among sleep evaluation methods or according to the definition of short sleep (figure 4B). The association between short sleep and mortality from all causes was significant only in studies conducted in North America. We were unable to perform the analysis by age subgroups, once one study ${ }^{40}$ included only elderly aged [more than equal to symbol] 80 years and other studies did not provide analysis by age groups.

Table 4 shows estimates, obtained from random effects models, for sleep duration and all-cause mortality according to the variables included in the multivariate analysis. The inclusion of covariates resulted in the attenuation of the association between long sleep duration and mortality, with moderate heterogeneity among studies.

\section{Risk of bias}

In most of the included studies, sleep duration was selfreported and this method does not differentiate time asleep from time in bed. Although we have included risk assessments carried out in multivariate analyses, three studies did not provide adjusted analyses, ${ }^{29} 3344$ one study adjusted only for age, ${ }^{36}$ and for other studies, this was done for a few confounders. ${ }^{42} 4647$ In an attempt to control for confounding, additional analyses were carried out including studies which had similar adjustment for confounding factors. Nevertheless, adjustments for health conditions resulted in attenuation of the association between long sleep and mortality. Another limitation of the primary studies is the lack of assessment of sleep quality, which can interfere with sleep duration.

\section{DISCUSSION}

To the best of our knowledge, this is the first systematic review with meta-analysis that investigated the relationship of sleep duration with mortality in the elderly population. Pooled analyses of data from 29 cohort studies indicate that sleep duration exceeding 8,9 or $10 \mathrm{~h}$, compared with an average sleep duration of $7-8 \mathrm{~h}$, was associated with an approximately 33\% increased risk of all-cause mortality. Additionally, data from eight cohorts provided an estimated $43 \%$ increased risk for 
A LONG SLEEP

Sleep duration assessment

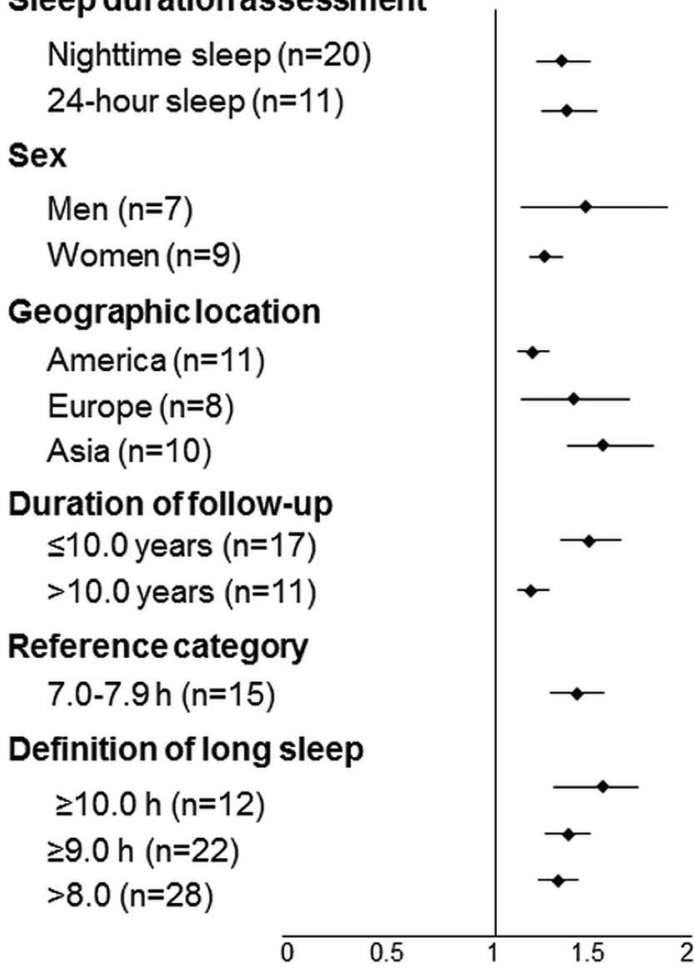

Relative Risk (95\% Cl) I² statistic (\%) P-value

$\begin{array}{lll}1.33(1.21-1.47) & 61.0 & \mathrm{P}<0.001 \\ 1.36(1.23 .1 .51) & 61.2 & \mathrm{P}=0.004 \\ & & \\ 1.45(1.13-1.86) & 79.8 & \mathrm{P}<0.001 \\ 1.25(1.17-1.34) & 65.6 & \mathrm{P}=0.003 \\ & & \\ 1.19(1.11-1.27) & 26.4 & \mathrm{P}=0.192 \\ 1.39(1.13-1.67) & 46.5 & \mathrm{P}=0.070 \\ 1.54(1.36-1.79) & 53.5 & \mathrm{P}=0.022 \\ & & \\ 1.47(1.33-1.63) & 39.4 & \mathrm{P}=0.048 \\ 1.18(1.11-1.27) & 45.9 & \mathrm{P}=0.048 \\ & & \\ 1.41(1.28-1.54) & 65.0 & \mathrm{P}<0.001 \\ & & \\ 1.56(1.32-1.74) & 78.0 & \mathrm{P}<0.001 \\ 1.39(1.28-1.50) & 67.5 & \mathrm{P}<0.001 \\ 1.34(1.24-1.44) & 63.3 & \mathrm{P}<0.001\end{array}$

B SHORT SLEEP

Sleep duration assessment

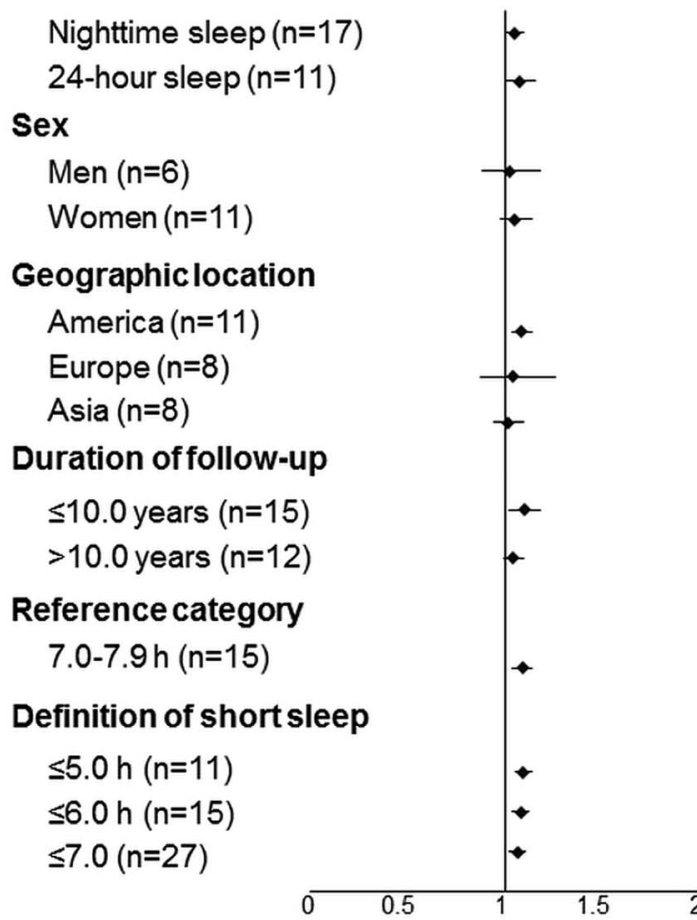

Relative Risk (95\% IC) I² statistic (\%) P-value

$\begin{array}{lll}1.06(1.01-1.10) & 0.0 & P=0.554 \\ 1.08(1.01 .1 .16) & 25.2 & P=0.204 \\ & & \\ 1.03(0.89-1.19) & 34.7 & P=0.176 \\ 1.06(0.98-1.15) & 0.0 & P=0.488 \\ & & \\ 1.09(1.04-1.15) & 0.0 & P=0.868 \\ 1.05(0.88-1.27) & 44.9 & P=0.080 \\ 1.02(0.95-1.10) & 2.4 & P=0.411 \\ 1.11(1.03-1.19) & 0.0 & P=0.554 \\ 1.05(1.00-1.10) & 17.9 & P=0.268 \\ & & \\ 1.10(1.04-1.15) & 4.8 & P=0.398 \\ 1.10(1.06-1.15) & 0.0 & P=0.478 \\ 1.09(1.04-1.13) & 0.0 & P=0.698 \\ 1.07(1.03-1.11) & 4.7 & P=0.395\end{array}$

Figure 4 Subgroup analyses to explore sources of heterogeneity in (A) long and (B) short sleep duration.

cardiovascular mortality in the long sleep duration group. Similar analysis for short sleep duration $(<7 \mathrm{~h})$ extracted from 27 cohort studies also showed a statistically significant association with all-cause mortality, with a $6 \%$ higher risk. Statistically significant association with mortality was not observed for long daytime sleep $(>2 \mathrm{~h})$. 
Table 4 Analysis of subgroups for the association between sleep duration and all-cause mortality according to the models of multivariate analysis carried out for control of confounding factors in the individual studies

\begin{tabular}{|c|c|c|c|c|}
\hline & Number of cohorts & Relative risk $(95 \% \mathrm{Cl})$ & $\mathrm{I}^{2}$ statistic (\%) & $\begin{array}{l}Q \text { test } \\
\text { p Value }\end{array}$ \\
\hline \multicolumn{5}{|l|}{ Short sleep } \\
\hline Unadjusted & 15 & 1.07 (0.96 to 1.20$)$ & 78.2 & $<0.001$ \\
\hline Model 1† & 9 & $1.17(1.00$ to 1.36$)$ & 43.2 & 0.080 \\
\hline Model $2 \ddagger$ & 4 & $1.20(0.92$ to 1.58$)$ & 66.4 & 0.030 \\
\hline Model 3§ & 7 & 1.10 (0.99 to 1.23$)$ & 0.0 & 0.587 \\
\hline Model 4ף & 18 & 1.06 (1.00 to 1.13$)$ & 22.6 & 0.186 \\
\hline \multicolumn{5}{|l|}{ Long sleep } \\
\hline Unadjusted & 16 & $1.58(1.33 \text { to } 1.88)^{*}$ & 94.7 & $<0.001$ \\
\hline Model 1† & 11 & $1.67(1.36 \text { to } 2.05)^{*}$ & 76.2 & $<0.001$ \\
\hline Model $2 \ddagger$ & 5 & $1.80(1.47 \text { to } 2.12)^{*}$ & 52.6 & 0.077 \\
\hline Model $3 \S$ & 9 & $1.68(1.47 \text { to } 1.93)^{*}$ & 21.3 & 0.261 \\
\hline Model 4ף & 18 & $1.35(1.25 \text { to } 1.45)^{*}$ & 49.1 & 0.010 \\
\hline
\end{tabular}

There is a particular need to analyse the association between sleep duration and health outcomes in the elderly population. Sleep physiology undergoes significant changes across the lifespan and the distributions of sleep duration vary with age ${ }^{55}{ }^{56}$ Sleep disorders, including sleep apnoea, insomnia and periodic limb movement disorders, are prevalent in older individuals; each may impact sleep quality and duration. ${ }^{57} 58$ Numerous health problems prevalent in the elderly may also influence sleep duration or may influence physiological responses to altered sleep. ${ }^{55}$ For these reasons, the relationship between sleep duration and mortality may differ for older compared with middle-aged and younger adults.

Studies conducted in young individuals have identified a U-shaped relationship between sleep duration and allcause mortality. ${ }^{20} 21$ The association was weaker for short in comparison to long sleep duration. A meta-analysis conducted among adults and the elderly population showed an increased risk of dying for both short and long sleep duration. For short sleep duration, the magnitude of the effect was greater than the observed risk in this meta-analysis, but for long sleep duration the risk was similar. ${ }^{21}$ Our findings are in accordance with such studies, including similar estimates regarding the association of long sleep duration and cardiovascular mortality in the adult population. ${ }^{21}$ It has been postulated that the adverse health outcomes associated with prolonged sleep might be a consequence of underlying diseases, frailty and worse health status, or be a part of the dying process. ${ }^{19} 5960$ In addition, an increase in sleep duration that occurs during the last few weeks or months of life ${ }^{61}$ might be a consequence of the disease and not its cause.

Castro-Costa et $a l^{24}$ addressed reverse causality by repeating their analyses after excluding mortality data in the 2 years following the baseline assessment and observed similar results. Moreover, Mesas et $a \bar{l}^{4}$ found that long sleep duration was associated with greater mortality, even in those with better health status and with no cognitive impairment, depression or functional limitations. These findings suggest that mortality associated with long sleep duration is not entirely due to a worse health status, and that long sleep duration itself may be an independent predictor. Several mechanisms have been suggested to explain the association between long sleep and mortality, including sleep fragmentation and sympathetic nervous system activation, ${ }^{25} 6263$ changes in cytokine levels ${ }^{64}{ }^{65}$ and physiological reduction of the photoperiod (ratio of daylight to darkness), ${ }^{66} 67$ among others. $^{68-70}$ However, there is scarce solid evidence ${ }^{64}$ and long sleep duration could be a marker of physiological deviation from normal ageing. ${ }^{46}$ Nevertheless, if these mechanisms actually participate in the axis of causality, they may be linked and not mutually exclusive. ${ }^{25}$

In addition to residual confounding, misclassification of sleep duration should be considered as an alternative explanation. Sleep duration did not allow one to differentiate time asleep from time in bed-unless explicitly built as additional questions. ${ }^{46}$ Prolonged time in bed per se may be harmful through metabolic effects including insulin resistance and deconditioning. ${ }^{25} 63$ While actigraphy can provide objective measures of sleep duration, ${ }^{71}$ it also has its limitations and may systematically misclassify sleep duration in individuals with sleep apnoea, depression and neurological disorders. Moreover, sleep duration was assessed at one point in time in all studies, and a single measurement of exposure may not capture the sustained effects of sleep duration over time, ${ }^{8}$ mostly if the reported period was shortly before death. ${ }^{37}$ 
Although there are several cut-offs for the duration of sleep, we did not have a hypothesis a priori on a specific cut-off for short or long sleep. The decisions taken before starting the analysis were to analyse the extreme cut-offs for long and short sleep, where several cut-off points were available, keeping the reference category as presented in the individual articles. Recognising the diversity of cut-offs, subgroup analyses were performed using all available cut-off points. All cut-offs for long sleep duration were associated with mortality, but there was no association with the cut-offs for short sleep duration.

The pooled effect sizes seem small, but evaluation of health issues in the general population, compared with an individual study cohort, requires a different interpretation-even small shifts are of significant population value. $^{72}$ Data were analysed using the random effects model, resulting in more conservative summary estimates, in comparison to the fixed effect model. However, these analyses are likely to be fully generalised. ${ }^{73}$ Furthermore, the direction of the associations is consistent in different populations, allowing one to assume that they are not dependent of lifestyles and hours of exposure to the sun, which influence sleep duration. ${ }^{8} 74$ Although meta-analysis detected significant heterogeneity among studies, further sensitivity analysis was in favour of consistent effects across populations.

\section{Study limitations}

There are limitations with using these findings to infer that long sleep duration is detrimental to the health of elderly individuals. The quality of the data cannot go beyond the quality of the individual studies included. ${ }^{75}$ A meta-analysis of observational data, though prospective, cannot fully control for confounding factors. ${ }^{8}$ Geriatric syndromes, including functional disability, immobility, frailty, cognitive impairment-especially dementia and depression-and use of sleep, antidepressant or antipsychotic medications predict mortality in older persons ${ }^{46} 7677$ and could also be associated with long sleep duration. ${ }^{64} 7879$ In most of the studies, these covariates were not included as potential confounding factors. Sleep quality remains a hypothesis to be addressed in future research.

The evidence of publication bias in the long sleep meta-analysis means that the results can only be representative of the studies that have been included and are unable to provide a representative inference of all studies published, but not included. ${ }^{8}$ Given the updated extensive search of articles, the use of conservative random effect models, and the results of subgroup and sensitivity analyses, it is unlikely that any addition to the reviewed studies would have generated summary estimates outside the reported $95 \%$ CIs, which are supported by the results of the trim and fill method.

Finally, studies included in this review used different categorical cut-off points in the analyses, some of them considering nighttime sleep and others $24 \mathrm{~h}$ sleep (including daytime sleep), hence precluding our ability to examine the associations between sleep duration and mortality at individual hour or smaller sleep duration categories. In this meta-analysis, long sleep duration did not include napping, but daytime included. In two meta-analysis published recently, statistically significant associations were found with all-cause mortality, but in a limited number of studies. ${ }^{80}{ }^{81}$ While the inconsistency reduces the generalisability of the conclusions, the convergence of findings strengthens the reliability of the association between long sleep duration and mortality. We attempted to combine categories into overall short and long sleep categories and, in subgroup analysis, we performed separate analysis according to the cut-point used and sleep duration assessment $(24 \mathrm{~h}$ sleep or nighttime).

\section{CONCLUSIONS}

This meta-analysis indicated that both long and short sleep duration are significantly associated with all-cause mortality in elderly individuals. Additionally, long sleep duration was also significantly associated with an increased risk for cardiovascular mortality. These findings suggest that sleep duration can be regarded as an additional behavioural risk factor or an adverse prognosis marker. The results must be considered in the context of the inherent limitations of observational studies, related to misclassification of sleep duration and potential residual confounding, especially due to geriatric syndromes. Future studies should be designed to address if modifying sleep duration could produce changes in health outcomes and mortality.

\section{Author affiliations}

${ }^{1}$ Postgraduate Studies Program in Epidemiology, School of Medicine, Universidade Federal do Rio Grande do Sul (UFRGS), Porto Alegre, Rio Grande do Sul, Brazil

${ }^{2}$ Postgraduate Studies Program in Cardiology, School of Medicine, Universidade Federal do Rio Grande do Sul, Porto Alegre, Rio Grande do Sul, Brazil

${ }^{3}$ Division of Cardiology, National Institute for Health Technology Assessment (IATS/CNPq), Hospital de Clinicas de Porto Alegre, Porto Alegre, Rio Grande do Sul, Brazil

${ }^{4}$ Department of Medicine, Sleep Medicine, Brigham and Women's Hospital, Boston, Massachusetts, USA

Contributors AAdS, FDF and SCF conceived the study aims and design. AAdS, CWS and RGBdM contributed to the systematic review and data extraction, performed the analysis, interpreted the results, and drafted the manuscript. RGBdM, SCF and FDF contributed to the revision of the data extraction, interpretation of results, and to the review of the manuscript. FDF and SR were important contributors to the interpretation and presentation of the manuscript. All the authors reviewed and approved the final version of the manuscript. SCF is the guarantor.

Funding This study was partially supported by the National Council for Scientific and Technological Development (CNPq), Brazil and Postgraduate Studies Program in Epidemiology, School of Medicine, Universidade Federal do Rio Grande do Sul (UFRGS). SCF and FDF have research scholarships from the CNPq, Brazil.

Disclaimer CNPq and HCPA did not take part in the study design, analysis, results interpretation or drafting of the manuscript. 
Competing interests None declared.

Provenance and peer review Not commissioned; externally peer reviewed.

Data sharing statement No additional data are available.

Open Access This is an Open Access article distributed in accordance with the Creative Commons Attribution Non Commercial (CC BY-NC 4.0) license, which permits others to distribute, remix, adapt, build upon this work noncommercially, and license their derivative works on different terms, provided the original work is properly cited and the use is non-commercial. See: http:// creativecommons.org/licenses/by-nc/4.0/

\section{REFERENCES}

1. Cappuccio FP, Stranges S, Kandala NB, et al. Gender-specific associations of short sleep duration with prevalent and incident hypertension: the Whitehall II Study. Hypertension 2007:50:693-700.

2. Cappuccio FP, Cooper D, D'Elia L, et al. Sleep duration predicts cardiovascular outcomes: a systematic review and meta-analysis of prospective studies. Eur Heart J 2011;32:1484-92.

3. Gangwisch JE, Heymsfield SB, Boden-Albala B, et al. Short sleep duration as a risk factor for hypertension: analyses of the First National Health and Nutrition Examination Survey. Hypertension 2006;47:833-9.

4. Gottlieb DJ, Redline S, Nieto FJ, et al. Association of usual sleep duration with hypertension: the Sleep Heart Health Study. Sleep 2006:29:1009-14

5. Ikehara S, Iso H, Date C, et al. Association of sleep duration with mortality from cardiovascular disease and other causes for Japanese men and women: the JACC study. Sleep 2009;32:295-301.

6. Meisinger $\mathrm{C}$, Heier M, Löwel $\mathrm{H}$, et al. Sleep duration and sleep complaints and risk of myocardial infarction in middle-aged men and women from the general population: the MONICA/KORA Augsburg Cohort Study. Sleep 2007;30:1121-7.

7. Ayas NT, White DP, Manson JE, et al. A prospective study of sleep duration and coronary heart disease in women. Arch Intern Med 2003:163:205-9.

8. Cappuccio FP, D'Elia L, Strazzullo P, et al. Quantity and quality of sleep and incidence of type 2 diabetes: a systematic review and meta-analysis. Diabetes Care 2010;33:414-20.

9. Gottlieb DJ, Punjabi NM, Newman AB, et al. Association of sleep time with diabetes mellitus and impaired glucose tolerance. Arch Intern Med 2005;165:863-7.

10. Van Cauter E, Holmback U, Knutson K, et al. Impact of sleep and sleep loss and neuroendocrine and metabolic function. Horm Res 2007;67(Suppl 1):2-9.

11. Cappuccio FP, Taggart FM, Kandala NB, et al. Meta-analysis of short sleep duration and obesity in children, adolescents and adults. Sleep 2008;31:619-26.

12. Gangwisch JE, Malaspina D, Boden-Albala B, et al. Inadequate sleep as a risk factor for obesity: analyses of the NHANES I. Sleep 2005;28:1289-96.

13. Hasler G, Buysse DJ, Klaghofer R, et al. The association between short sleep duration and obesity in young adults: a 13-year prospective study. Sleep 2004;27:661-6.

14. Singh M, Drake CL, Roehrs T, et al. The association between obesity and short sleep duration: a population-based study. Clin Sleep Med 2005;1:357-63.

15. Stranges S, Cappuccio FP, Kandala NB, et al. Cross-sectional versus prospective associations of sleep duration with changes in relative weight and body fat distribution: the Whitehall II Study. Am J Epidemiol 2008;167:321-9.

16. Steptoe A, Peacey V, Wardle J. Sleep duration and health in young adults. Arch Intern Med 2006;166:1689-92.

17. Ferrie JE, Shipley MJ, Cappuccio FP, et al. A prospective study of change in sleep duration: associations with mortality in the Whitehall II cohort. Sleep 2007;30:1659-66.

18. Hublin C, Partinen M, Koskenvuo M, et al. Sleep and mortality: a population-based 22-year follow-up study. Sleep 2007;30:1245-53.

19. Kripke DF, Garfinkel L, Wingard DL, et al. Mortality associated with sleep duration and insomnia. Arch Gen Psychiatry 2002;59:131-6.

20. Cappuccio FP, D'Elia L, Strazzullo $P$, et al. Sleep duration and all-cause mortality: a systematic review and meta-analysis of prospective studies. Sleep 2010;33:585-92.

21. Gallicchio L, Kalesan B. Sleep duration and mortality: a systematic review and meta-analysis. J Sleep Res 2009;18:148-58.
22. World Health Organization (WHO). Ageing and life course. 2012 [cited 11 Apr 2013]. http://www.who.int/ageing/about/facts/en/index. $\mathrm{html}$

23. Foley DJ, Monjan AA, Brown SL, et al. Sleep complaints among elderly persons: an epidemiologic study of three communities. Sleep 1995;18:425-32.

24. Castro-Costa E, Dewey ME, Ferri CP, et al. Association between sleep duration and all-cause mortality in old age: 9-year follow-up of the Bambuí Cohort Study, Brazil. J Sleep Res 2011;20:303-10.

25. Grandner MA, Drummond SP. Who are the long sleepers? Towards an understanding of the mortality relationship. Sleep Med Rev 2007;11:341-60.

26. Moher D, Liberati A, Tetzlaff J, et al. The PRISMA Group (2009). Preferred Reporting Items for Systematic Reviews and Meta-Analyses: the PRISMA statement. BMJ 2009;339:b2535.

27. von Elm E, Altman DG, Egger M, et al. The Strengthening the Reporting of Observational Studies in Epidemiology (STROBE) statement: guidelines for reporting observational studies. Epidemiology 2007;18:800-4.

28. Duval S, Tweedie R. Trim and fill: a simple funnel-plot-based method of testing and adjusting for publication bias in meta-analysis. Biometrics 2000;56:455-63.

29. Kronholm E, Laatikainen T, Peltonen M, et al. Self-reported sleep duration, all-cause mortality, cardiovascular mortality and morbidity in Finland. Sleep Med 2011;12:215-21.

30. Mallon L, Broman JE, Hetta J. Sleep complaints predict coronary artery disease mortality in males: a 12-year follow-up study of a middle-aged Swedish population. J Intern Med 2002;251:207-16.

31. Kakizaki M, Kuriyama S, Nakaya N, et al. Long sleep duration and cause-specific mortality according to physical function and self-rated health: the Ohsaki Cohort Study. J Sleep Res 2013;22:209-16.

32. Zuurbier LA, Luik AI, Hofman A, et al. Fragmentation and stability of circadian activity rhythms predict mortality: the Rotterdam study. Am J Epidemiol 2015;181:54-63.

33. Kripke DF, Langer RD, Elliott JA, et al. Mortality related to actigraphic long and short sleep. Sleep Med 2011;12:28-33.

34. Stone KL, Ewing SK, Ancoli-Israel S, et al. Self-reported sleep and nap habits and risk of mortality in a large cohort of older women. J Am Geriatr Soc 2009;57:604-11.

35. Ensrud KE, Blackwell TL, Ancoli-Israel S, et al. Sleep disturbances and risk of frailty and mortality in older men. Sleep Med 2012:13:1217-25

36. Burazeri G, Gofin J, Kark JD. Siesta and mortality in a Mediterranean population: a community study in Jerusalem. Sleep 2003;26:578-84.

37. Lan TY, Lan TH, Wen CP, et al. Nighttime sleep, Chinese afternoon nap, and mortality in the elderly. Sleep 2007;30:1105-10.

38. Lee JS, Auyeung TW, Leung J, et al. Long sleep duration is associated with higher mortality in older people independent of frailty: a 5-year cohort study. J Am Med Dir Assoc 2014;15:649-54.

39. Jung KI, Song $\mathrm{CH}$, Ancoli-Israel $\mathrm{S}$, et al. Gender differences in nighttime sleep and daytime napping as predictors of mortality in older adults: the Rancho Bernardo Study. Sleep Med 2013;14:12-19.

40. Werle MH, Moriguchi E, Fuchs SC, et al. Risk factors for cardiovascular disease in the very elderly: results of a cohort study in a city in southern Brazil. Eur J Cardiovasc Prev Rehabil 2011;18:369-77.

41. Suzuki E, Yorifuji T, Ueshima K, et al. Sleep duration, sleep quality and cardiovascular disease mortality among the elderly: a population-based cohort study. Prev Med 2009;49:135-41.

42. Kaplan GA, Seeman TE, Cohen RD, et al. Mortality among the elderly in the Alameda County Study: behavioral and demographic risk factors. Am J Public Health 1987;77:307-12.

43. Gale C, Martyn C. Larks and owls and health, wealth, and wisdom. BMJ 1998;317:1675-7.

44. Branch LG, Jette AM. Personal health practices and mortality among the elderly. Am J Public Health 1984;74:1126-9.

45. Gangwisch JE, Heymsfield SB, Boden-Albala B, et al. Sleep duration associated with mortality in elderly, but not middle-aged, adults in a large US sample. Sleep 2008;31:1087-96.

46. Rumble R, Morgan K. Hypnotics, sleep, and mortality in elderly people. J Am Geriatr Soc 1992;40:787-91.

47. Ruigomez A, Alonso J, Anto JM. Relationship of health behaviours to five-year mortality in an elderly cohort. Age Ageing 1995;24:113-19.

48. Cohen-Mansfield J, Perach R. Sleep duration, nap habits, and mortality in older persons. Sleep 2012;35:1003-9.

49. Kim Y, Wilkens LR, Schembre SM, et al. Insufficient and excessive amounts of sleep increase the risk of premature death from cardiovascular and other diseases: the Multiethnic Cohort Study. Prev Med 2013;57:377-85. 
50. Seki N. Relationships between walking hours, sleeping hours, meaningfulness of life (ikigai) and mortality in the elderly: prospective cohort study. Nihon Eiseigaku Zasshi 2001;:56:535-40.

51. Leng Y, Wainwright NW, Cappuccio FP, et al. Daytime napping and the risk of all-cause and cause-specific mortality: a 13-year follow-up of a British population. Am J Epidemiol 2014;179:1115-24.

52. Yeo Y, Ma SH, Park SK, et al. A prospective cohort study on the relationship of sleep duration with all-cause and disease-specific mortality in the Korean Multi-center Cancer Cohort study. J Prev Med Public Health 2013;46:271-81.

53. Chen HC, Su TP, Chou P. A nine-year follow-up study of sleep patterns and mortality in community-dwelling older adults in Taiwan. Sleep 2013;36:1187-98.

54. Mesas AE, López-García E, León-Muñoz LM, et al. Sleep duration and mortality according to health status in older adults. J Am Geriatr Soc 2010;58:1870-7.

55. Fragoso CAV, Gill TM. Sleep complaints in community-living older persons: a multifactorial geriatric syndrome. J Am Geriatr Soc 2007;55:1853-66.

56. Ohayon MM, Carskadon MA, Guilleminaut C, et al. Meta-analysis of quantitative sleep parameters from childhood to old age in healthy individuals: developing normative sleep values across the human lifespan. Sleep 2004:27:1255-73.

57. Ancoli-Israel S. Sleep and its disorders in aging populations. Sleep Med 2009;10(Suppl 1):S7-11.

58. Crowley K. Sleep and sleep disorders in older adults. Neuropsychol Rev 2011;21:41-53.

59. Burazeri G, Gofin J, Kark JD. Over 8 hours of sleep-marker of increased mortality in Mediterranean population: follow-up population study. Croat Med J 2003;44:193-8.

60. Bliwise DL, King AC, Harris RB. Habitual sleep durations and health in a 50-65-year-old population. J Clin Epidemiol 1994;47:35-41.

61. Teno JM, Weitzen S, Fennell ML, et al. Dying trajectory in the last year of life: does cancer trajectory fit other diseases? J Palliative Med 2001;4:457-64.

62. Chien KL, Chen PC, Hsu HC, et al. Habitual sleep duration and insomnia and the risk of cardiovascular events and all-cause death: report from a community-based cohort. Sleep 2010;33:177-84.

63. Youngstedt SD, Kripke DF. Long sleep and mortality: rationale for sleep restriction. Sleep Med Rev 2004;8:159-74.

64. Papanicolaou DA, Wilder RL, Manolagas SC, et al. The pathophysiologic roles of interleukin-6 in human disease. Ann Intern Med 1998;128:127-37.

65. Rogers NL, Szuba MP, Staab JP, et al. Neuroimmunologic aspects of sleep and sleep loss. Semin Clin Neuropsychiatry 2001;6:295-307.

66. Aujard F, Dkhissi-Benyahya O, Fournier I, et al. Artificially accelerated aging by shortened photoperiod alters early gene expression (FOS) in the suprachiasmatic nucleus and sulfatoxymelatonin excretion in a small primate, Microcebus murinus. Neuroscience 2001;105:403-12.

67. Gordon SH, Tucker SA. Effect of photoperiod and daily food access time on mortality and performance of male broilers. Br Poult Sci 1998;39(Suppl):S11-12.

68. Hartmann E, Baekeland F, Zwilling GR. Psychological differences between long and short sleepers. Arch Gen Psychiatry 1972;26:463-8.

69. Grandner MA, Kripke DF. Self-reported sleep complaints with long and short sleep: a nationally representative sample. Psychosom Med 2004;66:239-41.

70. Minois N. Longevity and aging: beneficial effects of exposure to mild stress. Biogerontology 2000;1:15-29.

71. Lauderdale DS, Knutson KL, Yan LL, et al. Self-reported and measured sleep duration: how similar are they? Epidemiology 2008;19:838-45.

72. Young T. Increasing sleep duration for a healthier (and less obese?) population tomorrow. Sleep 2008;31:593-4.

73. Borenstein M, Hedges LV, Higgins JPT, et al. Introduction to meta-analysis. Jonh Wiley \& Sons Ltd, 2009.

74. Bliwise DL. Invited commentary: cross-cultural influences on sleepbroadening the environmental landscape. Am J Epidemiol 2008;168:1365-6.

75. Downs SH, Black N. The feasibility of creating a checklist for the assessment of the methodological quality both of randomised and non-randomised studies of health care interventions. $J$ Epidemiol Community Health 1998;52:377-84.

76. Anpalahan M, Gibson SJ. Geriatric syndromes as predictors of adverse outcomes of hospitalization. Intern Med J 2008;38:16-23.

77. Kane RL, Shamliyan T, Talley $\mathrm{K}$, et al. The association between geriatric syndromes and survival. J Am Geriatr Soc 2012;60:896-904

78. Patel SR, Malhotra A, Gottlieb DJ, et al. Correlates of long sleep duration. Sleep 2006;29:881-9.

79. Stranges S, Dorn JM, Shipley MJ, et al. Correlates of short and long sleep duration: a cross-cultural comparison between the United Kingdom and the United States: the Whitehall II Study and the Western New York Health Study. Am J Epidemiol 2008;168:1353-64.

80. Zhong G, Wang Y, Tao T, et al. Daytime napping and mortality from all causes, cardiovascular disease, and cancer: a metaanalysis of prospective cohort studies. Sleep Med 2015;16: 811-19.

81. Liu X, Zhang Q, Shang X. Meta-analysis of self-reported daytime napping and risk of cardiovascular or all-cause mortality. Med Sci Monit 2015;21:1269-75. 